\title{
Using network dynamic fMRI for detection of epileptogenic foci
}

\author{
Sanja Nedic ${ }^{1,2}$, Steven M. Stufflebeam², Carlo Rondinoni ${ }^{3}$, Tonicarlo R. Velasco ${ }^{3}$, Antonio C. dos Santos ${ }^{3}$,
} Joao P. Leite ${ }^{3}$, Ana C. Gargaro ${ }^{3}$, Lilianne R. Mujica-Parodi ${ }^{1,2^{*}}$ and Jaime S. Ide $\mathrm{e}^{1,2,4^{*}}$

\begin{abstract}
Background: Epilepsy is one of the most prevalent neurological disorders. It remains medically intractable for about one-third of patients with focal epilepsy, for whom precise localization of the epileptogenic zone responsible for seizure initiation may be critical for successful surgery. Existing fMRI literature points to widespread network disturbances in functional connectivity. Per previous scalp and intracranial EEG studies and consistent with excessive local synchronization during interictal discharges, we hypothesized that, relative to same regions in healthy controls, epileptogenic foci would exhibit less chaotic dynamics, identifiable via entropic analyses of resting state fMRI time series.
\end{abstract}

Methods: In order to first validate this hypothesis on a cohort of patients with known ground truth, here we test individuals with well-defined epileptogenic foci (left mesial temporal lobe epilepsy). We analyzed voxel-wise resting-state fMRI time-series using the autocorrelation function (ACF), an entropic measure of regulation and feedback, and performed follow-up seed-to-voxel functional connectivity analysis. Disruptions in connectivity of the region exhibiting abnormal dynamics were examined in relation to duration of epilepsy and patients' cognitive performance using a delayed verbal memory recall task.

Results: ACF analysis revealed constrained (less chaotic) functional dynamics in left temporal lobe epilepsy patients, primarily localized to ipsilateral temporal pole, proximal to presumed focal points. Autocorrelation decay rates differentiated, with $100 \%$ accuracy, between patients and healthy controls on a subject-by-subject basis within a leave-one-subject out classification framework. Regions identified via ACF analysis formed a less efficient network in patients, as compared to controls. Constrained dynamics were linked with locally increased and long-range decreased connectivity that, in turn, correlated significantly with impaired memory (local left temporal connectivity) and epilepsy duration (left temporal - posterior cingulate cortex connectivity).

Conclusions: Our current results suggest that data driven functional MRI methods that target network dynamics hold promise in providing clinically valuable tools for identification of epileptic regions.

Keywords: Epilepsy, Seizure, fMRI, Power spectrum scale invariance, Autocorrelation function, Classification, Complexity, Chaos, Fractal

\footnotetext{
*Correspondence: Lilianne.Strey@stonybrook.edu; Jaime.Ide@stonybrook.edu

${ }^{1}$ Department of Biomedical Engineering, Stony Brook University School of

Medicine, Stony Brook, NY 11794, USA

Full list of author information is available at the end of the article
} 


\section{Background}

Epilepsy is one of the most prevalent neurological disorders, affecting approximately 50 million people worldwide [1]. It is characterized by seizures, resulting from abnormal transient change in the synchronized firing of neurons [2]. For one-third of patients with focal epilepsy, debilitating seizures persist despite antiepileptic drug therapy, leaving surgical resection of the suspected epileptogenic focal region as the most effective treatment option [3, 4]. Precise localization of the epileptogenic zone responsible for initiation and propagation of seizures, and its delineation from eloquent cortex, are crucial for successful surgery. However, current standard non-invasive surgical evaluation (looking for congruence of seizure semiology, abnormalities on structural MRI images and spikes in scalp EEG recordings) fails to identify an epileptic focus in approximately $40 \%$ of patients with drug resistant epilepsy [5]. The current gold standard for localization of focal regions includes identification of an epileptogenic zone on intracranial EEG recordings combined with postoperative seizure freedom following its resection; however, invasive pre-surgical workup carries additional risks and has been associated with complications in about $23 \%$ of patients [5]. Thus, the development of noninvasive techniques capable of accurately localizing epileptogenic regions on a subject-by-subject basis will be critical for improving surgical outcomes.

Multimodal studies, especially those utilizing simultaneous EEG-fMRI recordings, increasingly have been used to provide complementary information in presurgical work-up. EEG-fMRI allows mapping of hemodynamic changes related to seizure-related events, such as interictal discharges (IEDs). Spikes are manually detected in EEG data and, in traditional "spike-correlated" analysis, they are treated as zero-duration events, convolved with canonical hemodynamic response function (HRF), and included as regressors of interest in a General Linear Model (GLM) along with simultaneously acquired BOLD time series as dependent variables. fMRI maps related to IEDs often show multiple regions or "networks," rather than focal singularities, and thus effective connectivity approaches such as Dynamic Causal Modeling (DCM) have been proposed to identify which brain regions drive the generation of seizures within the epileptic network $[6,7]$.

The EEG-fMRI approach suffers from a few potential drawbacks. First, a significant portion of subjects do not experience enough detectable IEDs during simultaneous recording. This problem is partially addressed by introduction of topography-related techniques, which, instead of requiring simultaneously recorded spikes, use subjectspecific voltage maps based on long-term video monitoring [8]. Second, epilepsy may alter the shape of the HRF, which could result in decreased sensitivity [9]. Third, in addition to setup time, EEG-fMRI requires on average
30 min of motionless cooperation from subjects, which may be problematic for certain patients, especially children. Fourth, the use of scalp EEG is inherently constrained by its limited sensitivity to deep activity. Despite this, sensitivity of topography-related EEG-fMRI in refractory focal epilepsy was found to be about $80 \%[8,10]$. However, measured sensitivity appears to be highly studydependent. For example, a recent study proposing concurrent use of four different modalities found that topography-related EEG-fMRI method on its own showed clinically meaningful result in five out of twelve studied patients ([11]). Here we propose an alternative data-driven method utilizing only resting state fMRI data.

As a matter of general research strategy, any method with potential to identify seizure onset zones first needs to be validated with respect to a "ground truth." In the case of epilepsy, the most straightforward option is to use patients with clinically well-defined focal regions; after validation against these cases, one can then apply the method to more challenging cryptogenic cases. Mesial Temporal Lobe Epilepsy (MTLE) is the most prevalent and best-characterized subset of drug resistant focal epilepsy in adults, and thus is an ideal cohort for validation of novel methods aimed at identification and localization of epileptic foci and/or networks.

Brain regions involved in the onset and propagation of MTLE have been studied extensively, with the most commonly associated pathology being hippocampal sclerosis (HS). Seizures originating in the medial temporal region are known to rapidly spread to lateral temporal regions, the insula, the thalamus, and the contralateral temporal lobe (among other regions) [12], suggesting that MTLE may be characterized by a network disturbance. Indeed, recent structural and functional MRI studies have revealed widespread abnormalities, with structural changes primarily involving atrophy of ipsilateral temporal pole and other temporolimbic structures [13, 14]. Functional MRI (fMRI) studies have found changes in functional connectivity of the temporal region with other brain areas, along with impaired resting state networks such as perceptual, attention and default mode networks [15-24].

Graph theoretical network analyses of brain networks use sets of nodes connected by edges in order to quantify general structural features of brain connectivity between regions. Numerous studies suggest that healthy brain networks show a high degree of small-worldness (for a review see [25]), which provides a balance between local clustering (characteristic of highly ordered, regular networks) and long-range connections (characteristic of low order, random networks). This balance reflects the result of synaptic optimization over the efficiency of information transfer and the need for redundancy in case of injury. In individuals with focal temporal and extratemporal neocortical epilepsy, fMRI, EEG, MEG, and intracerebral 
recordings suggest that this balance appears to be shifted towards predominantly local clustering [20,26, 27], which may predispose the network towards synchronized oscillations characteristic of seizures [28].

However, findings are not clear-cut. While many studies report decreases in functional connectivity localized near the suspected seizure onset zone [16, 17, 22, 23], others show increases [15, 20, 29-31], potentially due to differences in connectivity metrics used, as well as heterogeneity of patient populations and small sample sizes. It is important to note that, in spite of the benefits of considering epileptic networks, accurate localization is still clinically desirable as suggested by the relatively high success rate of surgical treatment for 'focal' epilepsies (about $66 \%$ ) [2]. Yet most fMRI studies aimed at localization of the epileptogenic zone point to widespread abnormalities that are, at best, lateralized to one of the hemispheres.

Complex systems produce outputs that are balanced between overly chaotic and overly predictable dynamics; as such, they have the advantage of requiring a minimum of energy both to respond to inputs as well as to return to baseline (as required for allostasis). Complexity in brain activity has been observed and modeled on many levels, from neurotransmitter release [32], neuronal spiking [33, 34] and local field potentials [35] to slow cortical potentials [36], electrocorticography (ECoG) [37] and EEG [38], suggesting that scale-free behavior may be fundamental to efficient neural information processing. Deviations from optimal range of functioning in fMRI time series have been used diagnostically in identifying brain-based disease $[37,39,40]$. In the case of a disease as heterogeneous as epilepsy, fMRI's exploratory capability (simultaneously acquiring functional information over focal, hemispheric, and whole-brain neural networks) may provide a clinically valuable tool in guiding placement of intracranial EEG, as the process of seizure generation is not necessarily confined to a focal area and may involve distant or contralateral areas of the brain. Here we propose to use autocorrelation function (ACF) as a measure of complexity in resting-state fMRI time series, with the aim of localizing deviations from optimal dynamics in epilepsy.

Brain trauma increases risk for seizures. One possible mechanism suggested by animal models of epilepsy is the enhanced synaptic sprouting due to MMP-9 mediated matrix-degradation that occurs as a compensatory response to injury [41]. Alternatively, metabolic damage affecting the brain's ability to utilize glucose may impact the brain's creation of long-range, but not short-range, connections, upsetting balance between the two. Thus, we hypothesized that the hippocampal sclerosis common to MTLE patients might result in hubs surrounded by more dense local connectivity. This can constrain dynamics within the hub either by increased density of inhibitory or excitatory inputs, both of which would result in less chaotic resting state fMRI time series. We expected abnormal dynamics to be identifiable through comparison of decay rates of the time series' ACF in patients and in healthy controls. This is consistent with previous findings from scalp and intracranial EEG studies [42-45], as well as with excessive local synchronization during interictal discharges.

Less chaotic dynamics in focal region(s) may lead to increased local synchronization and transient seizures. Repeated seizures could in turn lead to damage in connections of this region with contralateral regions and major hubs in the brain. We therefore hypothesized that these disconnections would be detectable via seed-to-voxel functional connectivity analyses of fMRI time-series, using the region with abnormal dynamics (identified through ACF analyses) as a seed. Finally, we expected that abnormalities in connectivity would correlate with the duration of epilepsy as well as with patients' cognitive performance on a verbal memory recall task. To determine whether group differences achieving statistical significance continued to hold on the single-subject level (as required for future application of individual neurodiagnostics to other, cryptogenic, forms of epilepsy), we applied Gaussian Process Classification with leave-one-subject-out cross validation to ACF decay rates in regions with altered dynamics.

\section{Methods \\ Participants}

Nineteen patients (mean age $=40$ years $\pm 13 ; 8$ males, 11 females) with mesial left temporal lobe epilepsy (LTLE) were included in this study based on a clear clinical diagnosis of unilateral (left) temporal epileptic activity according to concordant clinical information. The inclusion criteria were: 1) seizure semiology consistent with MTLE, which included epigastric, autonomic, or psychic auras followed by behavioral arrest, progressive clouding of consciousness, oroalimentary and manual automatisms, and autonomic phenomena; 2) anterior and mesial temporal interictal spikes; 3) video-EEG monitoring with seizure onsets arising exclusively from the temporal lobe; 4) MRI with no other lesion than hippocampal atrophy and a hyperintense signal on T2-weighted sequences; and 5) medically refractory MTLE, defined as failure to respond to at least two antiepileptic drugs after adequate trials. For patients whose scalp ictal EEG recordings were inconclusive, foramen oval electrodes or depth electrodes were used to define lateralization of seizure foci. Table 1 lists demographic and clinical characteristics of all patients studied here. Nineteen healthy controls (mean age $=41$ years \pm 12; 8 males, 11 females), age and sex-matched to patients (2-sample $t$ test $p=0.78$ ), were scanned under the same protocol. Because temporal lobe epilepsy affects the hippocampus, and is associated with progressive memory 
Table 1 Demographic and clinical information

\begin{tabular}{|c|c|c|c|c|c|c|c|c|c|c|}
\hline Patient & Gender & Age/Onset & $\begin{array}{l}\text { Frequency } \\
\text { (month) }\end{array}$ & $\begin{array}{l}\text { Febrile } \\
\text { seizures }\end{array}$ & Seizure type & Interictal EEG & Ictal EEG & MRI findings & $\begin{array}{l}\text { Antiepileptic } \\
\text { medications }\end{array}$ & $\begin{array}{l}\text { Surgical } \\
\text { outcome }\end{array}$ \\
\hline 1 & $\mathrm{~F}$ & $44 / 18$ & 30 & No & Masticatory automatisms & Left temporal paroxysms & $\begin{array}{l}\text { Bilateral theta rhythm, late } \\
\text { left lateralization }\end{array}$ & Left HA & PHT, TPM & 3 \\
\hline 2 & M & $59 / 10$ & 4 & Yes & Complex partial & $100 \%$ left temporal & Left temporal & Left HA & CBZ & 1 \\
\hline 3 & $\mathrm{~F}$ & $27 / 13$ & 4 & No & Complex partial & $\begin{array}{l}70 \% \text { left, } 30 \% \text { right } \\
\text { temporal }\end{array}$ & Left temporal & Left HA & LTG, CLB, OXC & 1 \\
\hline 4 & M & $47 / 24$ & 4 & No & Lack of consciousness, no aura & $\begin{array}{l}\text { Left temporal sharp } \\
\text { waves }(89 \%, T 1,79)\end{array}$ & Left temporal theta & Left HA & CBZ, LTG, CLB & 1 \\
\hline 5 & $\mathrm{~F}$ & $32 / 19$ & 0.5 & No & Complex partial & Rare left temporal & Left temporal & Discrete left HA & $\mathrm{CBZ}, \mathrm{PB}, \mathrm{CLB}$ & 1 \\
\hline 6 & $\mathrm{~F}$ & $34 / 25$ & 4 & No & Complex partial & $\begin{array}{l}53 \% \text { left, } 47 \% \text { right } \\
\text { temporal }\end{array}$ & Left temporal & $\begin{array}{l}\text { Discrete left HA, } \\
\text { normal volume }\end{array}$ & TPM, OXC, CLB & 3 \\
\hline 7 & M & $26 / 5$ & 4 & No & Complex partial & Normal & Left temporal delta rhythm & Left HA & VPA, OXC, CLB & 1 \\
\hline 8 & $\mathrm{~F}$ & $44 / 9$ & 4 & No & Complex partial & $\begin{array}{l}70 \% \text { left, } 30 \% \text { right } \\
\text { temporal }\end{array}$ & Left temporal & Left HA & CBZ, TPM, CLN & 3 \\
\hline 9 & $\mathrm{~F}$ & $47 / 17$ & 4 & No & $\begin{array}{l}\text { Lack of consciousness, } \\
\text { automatisms }\end{array}$ & $\begin{array}{l}\text { Slow theta in left } \\
\text { temporal }\end{array}$ & $\begin{array}{l}\text { Bilateral theta activity followed } \\
\text { by left temporal theta }\end{array}$ & Left HA & LTG, CBZ & 1 \\
\hline 10 & M & $37 / 2$ & 2 & No & Complex partial & $\begin{array}{l}95 \% \text { left, } 5 \% \text { right } \\
\text { temporal }\end{array}$ & Left temporal & Left HA & OXC, LTG & 1 \\
\hline 11 & M & $46 / 18$ & $\mathrm{n} / \mathrm{a}$ & No & Complex partial & $\begin{array}{l}\text { Slow waves in left } \\
\text { temporal }\end{array}$ & $\begin{array}{l}\text { Left hemisphere diffuse } \\
\text { desynchronization }\end{array}$ & Left HA & PHT, PB & 1 \\
\hline 12 & $\mathrm{~F}$ & $52 / 14$ & 2 & Yes & Complex partial & $100 \%$ left temporal & Left temporal & Left HA & CBZ, TPM & 2 \\
\hline 13 & $\mathrm{~F}$ & $22 / 19$ & 3 & Yes & $\begin{array}{l}\text { Tonic-clonic secondarily } \\
\text { generalized }\end{array}$ & Left spikes & Left temporal & Left HA & OXC, VPA, CLB & 1 \\
\hline 14 & $\mathrm{~F}$ & $22 / 1$ & 16 & Yes & Complex partial & Left temporal & Left temporal & Left HA & TPM, OXC & 1 \\
\hline 15 & $\mathrm{~F}$ & $49 / 15$ & 1 & No & Complex partial & 100 \% left temporal & Left temporal & Left HA & $C B Z, P B$ & 1 \\
\hline 16 & M & $59 / 53$ & 1 & No & Complex partial & $100 \%$ left temporal & Left temporal & Left HA & PHT & 3 \\
\hline 17 & M & $20 / 4$ & 30 & Yes & $\begin{array}{l}\text { Epigastric aura and lack of } \\
\text { consciousness }\end{array}$ & $\begin{array}{l}\text { Left temporal slow } \\
\text { waves }\end{array}$ & Left temporal & Left HA & CBZ, VPA & 1 \\
\hline 18 & F & $30 / 10$ & 30 & No & $\begin{array}{l}\text { Complex partial seizures preceded } \\
\text { by sensitive auras }\end{array}$ & Left temporal theta rhythm & Complex partial seizure & $\begin{array}{l}\text { Discrete HA + } \\
\text { double cortex }\end{array}$ & LTG, CLB & $\mathrm{n} / \mathrm{a}$ \\
\hline 19 & M & 19/8 & 8 & No & Complex partial & $100 \%$ left temporal & Left temporal & Left HA & TPM, CBZ, CLN & 3 \\
\hline
\end{tabular}

Abbreviations: HA hippocampal atrophy, PHT Phenytoin, TPM Topiramate, CBZ Carbamazepine, LTG Lamotrigine, CLB Clobazam, OXC Oxcarbazepine, PB Phenobarbital, VPA Valproic Acid, CLN Clobazam. Surgical Outcome: 1 - seizure free, 2 - significant improvement, 3 - no improvement 
deficits [46], in order to assess clinical symptoms patients were asked to complete the Logical Memory Delayed Recall (LM-DR) subtest of the Wechsler Memory Scale Revised [47]. This task requires subjects to recall specific details of information presented orally in a story format thirty minutes after a single exposure. The research protocol was approved by the local Ethical Committee of Clinics Hospital at Ribeirao Preto, São Paulo, Brazil. Written informed consent was obtained from the patient for publication of their individual details in this manuscript. All subjects were older than 18 years of age and were capable of providing informed consent.

\section{Data acquisition}

All subjects were scanned using a Philips Achieva $3 \mathrm{~T}$ scanner with an eight-channel head coil. Whole brain functional volumes were acquired under resting state using soft-tone sequences $(\mathrm{TR}=2000 \mathrm{~ms}, \mathrm{TE}=30 \mathrm{~ms}$, 200 volumes, voxel dimensions: $3 \times 3 \times 4 \mathrm{~mm}, 32$ slices, $0.5 \mathrm{~mm}$ gap, matrix size $=80 \times 80$, flip angle $=80^{\circ}$ ). Subjects were instructed to keep their eyes open and to refrain from falling asleep. The eyes open condition was used rather than eyes closed condition to ensure that subjects remained awake during scanning. T1-weighted anatomical images were acquired using a conventional 3D-T1 MPRAGE sequence $(\mathrm{TR}=7.0, \mathrm{TE}=3.2$, matrix size $=240 \times 240$, flip angle $=8^{\circ}, 1 \mathrm{~mm}$ isotropic voxels).

\section{fMRI data preprocessing}

Functional MRI data were preprocessed by correcting for motion (rigid realignment, $6^{\circ}$-of-freedom), slice-time correction, normalization to MNI space (affine registration followed by a nonlinear transformation between average fMRI and EPI template, and sync interpolation), and smoothing with an 8-mm FWHM Gaussian kernel in SPM8 (www.fil.ion.ucl.ac.u/spm).

\section{Autocorrelation function (ACF) method}

An autocorrelation function (ACF) measures similarity (cross-correlation) of a signal with itself over different time lags, and can therefore be used to identify shifts towards chaos vs. order in the time domain, in a manner that is analogous to power spectral scale invariance (PSSI) analysis [40, 48, 49] in the frequency domain. PSSI and ACF are related via the Wiener-Khinchin theorem, which states that Fourier transform of the ACF is the power spectral density $\left(P(f)=\int_{-\infty}^{\infty} A C F(t) e^{-2 \pi i f t} d t\right)$.

PSSI of fMRI time series has already been used to quantify limbic dysregulation in trait anxious adults [49] and effectively discriminate between normal and pathogenic network dynamics in schizophrenia [40] and generalized anxiety disorder (DeDora et al., under review). PSSI analysis is based on the finding that fMRI BOLD time series exhibit power spectral density that follows a power law [50] $P(f) \sim 1 / f^{\beta}$, where $\beta$ is the scaling exponent. The scaling exponent $\beta$ then measures relative frequency content of the signal and is evaluated as the negative slope of a straight line fit to power spectral density as a function of frequency on log-log scale. Linear least squares method is employed. In contrast, the ACF of voxel-wise BOLD time series can be modeled as an exponential decay:

$$
A C F(t)=a e^{-b t}
$$

for which $t$ is time lag in units of TR, and $a$ and $b$ are constants such that $b>0$. The rate of this decay, which is proportional to the constant $b$, may be used as a compact measure of randomness of a time series, with higher $b$ signifying faster decay (more randomness) and lower $b$ signifying slower decay (more persistence). ACF $\mathrm{b}$ values are then related to the mean lifetime decay of a signal, $\tau$ (seconds), via the relation $\tau=1 / b \times T R$. Conceptually, $\tau$ represents the length of time that a signal maintains a high degree of association with its past values. A random time series (white noise) would have $\tau=0$. Larger values of $\tau$ would imply longer memory in the signal.

ACF has several advantages as compared to PSSI. First, ACF decay times have physiologically intuitive meaning, understood as self-similarity over different lengths of time (in seconds). Second, ACF model fits for fMRI time-series are improved compared with PSSI, because ACF avoids the need to use linear least squares fitting in log-log space [51].

Prior to whole brain voxel-wise computation of ACF b values, to remove potential effects of nuisance variables and improve signal to noise ratio, we performed further preprocessing of fMRI time series. The preprocessing procedure included detrending and regression of mean white matter and mean cerebrospinal fluid (CSF) signals, as well as regression of six degrees of motion. Motion parameters were derived from the realignment procedure, while global regressors were obtained from canonical masks for white matter and CSF included in the SPM8. To rule out head motion related artifacts on $\mathrm{b}$ values, we confirmed that there were no statistically significant differences in movement between the two subject groups using root mean square displacement (2 sample $t$-test: $p=0.37$ ). Root mean square displacement was calculated as the square root of the sum of squares of all six motion parameters [52].

The residual time series were band-pass filtered in the $0.01-0.1 \mathrm{~Hz}$ frequency range using 10th order Butterworth filter. Fitting was performed within a frequency range of $0.01-0.1 \mathrm{~Hz}$ since low frequency oscillations in this band show power-law behavior [37, 50], making PSSI-related inferences valid in this range, and are furthermore of special interest in resting-state fMRI 
connectivity analyses since they have been shown to have neuronal basis [51, 53, 54].

We estimated voxel-wise time series ACF using the Econometrics Toolbox implemented in MATLAB R2010a. We then fit an exponential function of the form $y=a e^{-b x}$ to the first $9(\mathrm{lag}=8)$ points of each voxel's ACF using the nonlinear least-squares fitting method as implemented in the Curve Fitting Toolbox, also part of MATLAB R2010a. Lag of 8 (16.8 s at TR of $2 \mathrm{~s}$ ) was determined as most appropriate based on considerations of goodness of fit of the exponential function and duration of canonical hemodynamic response [55].

Group differences in voxel-wise $b$ values were examined using a two sample $t$-test in SPM8. Clusters greater than 26 voxels with $p<0.005$ were isolated and used in further analyses. Prior to voxel-wise t-tests, the Lilliefors goodness-of-fit test (as implemented in REST toolbox [56]) was used to confirm that voxel-wise ACF-b values were normally distributed within each group of subjects. We found that the hypothesis of normality was supported by the vast majority (94\%) of brain voxels in each group at the $p=0.05$ level.

\section{Classification}

To verify that the group differences in b values were statistically significant and capable of differentiating between patients and healthy controls, even on an individual subject-by-subject basis, we conducted follow-up machine learning analyses on ACF b maps using Pattern Recognition for Neuroimaging Toolbox (PRoNTo) [57]. We constrained the analyses to those regions that were identified as having different $\mathrm{b}$ values between the two groups using the 2-sample $t$-test in SPM8. We employed Binary Gaussian Process Classification with leave one subject out cross-validation. Follow-up permutation testing (with 2000 repetitions) was used to test whether the obtained overall and class accuracies were significantly above chance, and thus indicate whether the pattern of ACF decay times in those brain regions encodes sufficient information to diagnose (correctly classify) MTLE.

\section{Functional connectivity}

In order to gain further understanding of the functional network features that might underlie abnormalities in fMRI signal dynamics, we used the cluster in the left anterior temporal pole identified as having most significantly different ACF b values in patients compared to controls (cluster extent $=59$ voxels; Fig. $1 \mathrm{a}$ ) as a seed in seed-tovoxel functional connectivity analysis using CONN Toolbox (http://www.nitrc.org/projects/conn/; v 13.p). We examined the differences in seed-to-voxel connectivity between LTLE patients and healthy controls using one-sided 2 sample t-tests. Time series were extracted from spatially smoothed images. They were detrended, despiked (a hyperbolic tangent squashing function was applied to reduce the influence of potential outlier scans), and white matter, cerebrospinal fluid, and motion parameters from SPM's realignment step and their first derivatives were regressed out as confounds. Finally, the residual time series were band-pass filtered in the [0.01-0.1] $\mathrm{Hz}$ range. Correlation maps were computed from the seed to all voxels in the brain and transformed to Fisher-z values (to ensure normality) prior to performing t-tests to look at differences between the two groups. We identified clusters of voxels that were significantly differently connected to the region exhibiting constrained dynamics (identified via $\mathrm{ACF}$ analysis) in patients relative to controls. We then used correlation analysis to test whether connectivity of these clusters to the seed was related to duration of epilepsy and verbal memory as measured by the LM-DR task.

\section{Graph-theoretic analyses}

To determine whether complexity and connectivity results reflected global differences in network structure, we used the CONN Toolbox to investigate graph-theoretic characteristics of the network with nodes at clusters identified as having different ACF b values in patients compared to controls. Bivariate correlation of the mean time series was used as a measure of association between the nodes. For this 16-node network, we compared the two subject groups with respect to Global Efficiency, Local Efficiency, Betweenness-Centrality, Average Path Length, Clustering Coefficient, and Degree under a range of values of cost $(0.1-0.3)[58,59]$.

\section{Results}

Left temporal lobe epilepsy patients show constrained dynamics of left temporal pole

Unbiased (exploratory) analyses identified the superior left temporal pole (peak MNI coordinate: $(-362-17)$, $p<0.005$ uncorrected, cluster extent $=59$ voxels, peak $\mathrm{T}_{36}=7.11$; Fig. 1) as showing the greatest differences between LTLE patients and healthy controls; patients showed significantly decreased ACF b values (i.e., slower ACF decay, more constrained dynamics). In addition, 15 other regions, all exceeding 27 voxels, showed lowered $\mathrm{ACF} \mathrm{b}$ values in patients at $p<0.005$. Regions involved include portions of the right inferior frontal gyrus, left thalamus, right precuneus, right caudate, bilateral heschl gyrus, bilateral inferior parietal and postcentral area, and bilateral insula. These results are presented in Table 2 . No regions showed increased ACF b values in patients compared to controls. We repeated the 2-sample $t$-test 38 times, each time leaving one subject out. Each time, a cluster at MNI (-36 2-17) was found to be significantly different between the LTLE patients and healthy controls, 


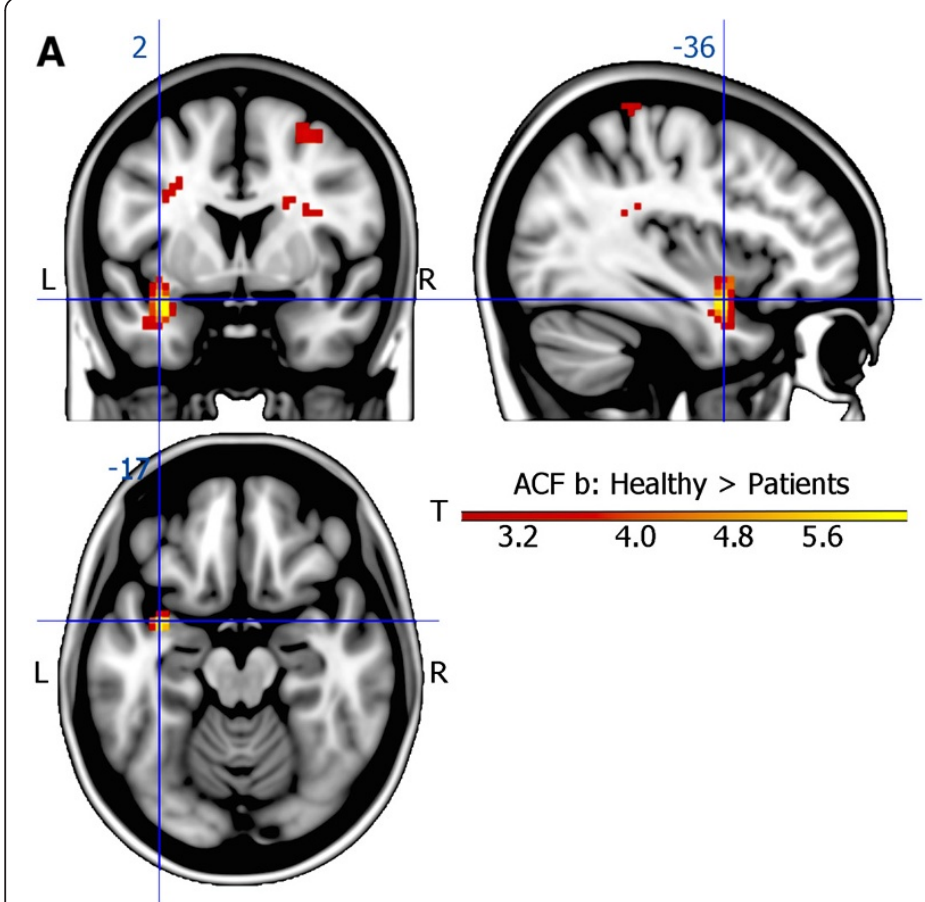

B

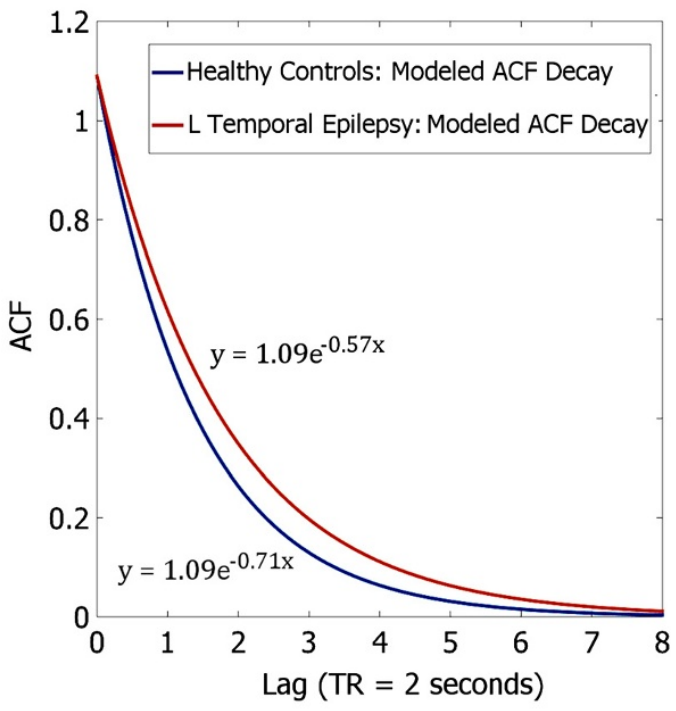

Fig. 1 Autocorrelation decay rates (ACF b) are altered close to presumed focal regions. a: Left Temporal Lobe Epilepsy (LTLE) patients exhibit lower ACF b values (slower decay of autocorrelation) relative to Healthy Controls $(\mathrm{HC})$ in the Left Superior Temporal Pole (peak MNI coordinate [-36, 2, -17$]$, cluster extent $=59$ voxels, peak $p=1.18 \times 10^{-8}, b_{\text {HC }}$ (cluster $)=0.71 \pm 0.09, b_{\text {LTLE }}($ cluster $\left.)=0.57 \pm 0.05\right)$ ). b: Modeled ACF decay for the left superior temporal pole cluster for HC and LTLE patients

Table $\mathbf{2}$ List of regions showing significantly different values of ACF b in patients relative to controls

\begin{tabular}{|c|c|c|c|c|}
\hline & \multicolumn{4}{|c|}{ Left TLE $<$ healthy controls } \\
\hline & Peak MNI & Location description (AAL) & Cluster extent & $t$-test (peak-level T $(p)$, \\
\hline 1 & {$[-36,2,-17]$} & L Superior Temporal Pole, Middle Temporal Pole, Insula, Amygdala & 59 & $7.11\left(1.18 \times 10^{-8}\right)$ \\
\hline 2 & {$[13,23,42]$} & R Inferior Frontal Gyrus, Pars Triangularis, Inferior Frontal Gyrus, Pars Opercularis & 71 & $4.59\left(2.61 \times 10^{-5}\right)$ \\
\hline 3 & {$[-27,-7,28]$} & L White Matter (Precentral) & 43 & $4.57\left(2.79 \times 10^{-5}\right)$ \\
\hline 4 & {$[-12,-10,-2]$} & L Thalamus & 33 & $4.56\left(2.85 \times 10^{-5}\right)$ \\
\hline \multirow[t]{2}{*}{5} & {$[-45,-37,43]$} & L Inferior Parietal Lobule, & 69 & $4.40\left(4.64 \times 10^{-5}\right)$ \\
\hline & & Postcentral Gyrus & & \\
\hline 6 & {$[8,21,25]$} & R Caudate & 69 & $4.31\left(5.95 \times 10^{-5}\right)$ \\
\hline 7 & {$[36,-22,19]$} & R Insula, R Heschl & 27 & $4.20\left(8.37 \times 10^{-5}\right)$ \\
\hline 8 & {$[30,-43,52]$} & R Inferior Parietal Lobule & 88 & $4.10\left(1.13 \times 10^{-4}\right)$ \\
\hline 9 & {$[15,-46,7]$} & R Precuneus & 34 & $4.07\left(1.22 \times 10^{-4}\right)$ \\
\hline \multirow[t]{2}{*}{10} & {$[-24,-34,70]$} & L Postcentral Gyrus, & 60 & $3.99\left(1.53 \times 10^{-4}\right)$ \\
\hline & & Paracentral Gyrus & & \\
\hline 11 & {$[30,41,1]$} & R White Matter (Frontal) & 42 & $3.89\left(2.01 \times 10^{-4}\right)$ \\
\hline 12 & {$[12,-112,-2]$} & L Calcarine & 31 & $3.84\left(2.41 \times 10^{-4}\right)$ \\
\hline 13 & {$[-30,-43,25]$} & L White Matter (Parietal) & 31 & $3.68\left(3.78 \times 10^{-4}\right)$ \\
\hline \multirow[t]{2}{*}{14} & {$[30,2,58]$} & R Middle Frontal Gyrus, & 29 & $3.62\left(4.46 \times 10^{-4}\right)$ \\
\hline & & Superior Frontal Gyrus & & \\
\hline 15 & {$[-54,-10,10]$} & L Heschl & 29 & $3.46\left(6.95 \times 10^{-4}\right)$ \\
\hline 16 & {$[3,-91,10]$} & L Calcarine & 30 & $3.28\left(1.20 \times 10^{-3}\right)$ \\
\hline
\end{tabular}

Two-sample $t$-test was performed in SPM8 $(p<0.005, \mathrm{k} \geq 27)$ 
confirming reliability across subjects, and the absence of outlier-effects. In addition, to probe the potential for single-subject analyses, we compared voxel-wise wholebrain individual patients' ACF $\mathrm{b}$ maps to the average healthy control ACF b map, and found that for 13 out of 19 patients there was a cluster $(>6$ voxels, median size $=$ 32 voxels) at the identical coordinate $(-362-17)$ which had $b$ values that were more than two standard deviations lower than those in the same region in the mean control map. Remaining patients had such clusters within the left temporal lobe.

\section{Classification analyses achieve $100 \%$ accuracy in distinguishing patients vs. controls}

To verify that the differences in ACF b values were not only statistically significant at the group level, but also capable of differentiating between patients and healthy controls even on a subject-by-subject basis, we conducted follow-up classification analyses on ACF b maps using Gaussian Process Classification with leave one subject out cross-validation. We used the mask consisting of 16 clusters identified as different between groups as a $2^{\text {nd }}$ level mask in PRoNTo. Every single subject was classified correctly, achieving $100 \%$ accuracy. Graph of prediction values and the associated density functions and weights map are shown in Fig. 2. While it is the combination of all weights that defines the model and individual contributions of voxels in the $2^{\text {nd }}$ level mask cannot be accessed directly, the weights map confirms that most discriminative voxels are the ones in the left temporal pole. Perfect accuracy implies that the b-values in these regions encode sufficient information for successful discrimination between the two groups.

Using only the top cluster, which showed most significant abnormality in patients according to the $t$-test, as a $2^{\text {nd }}$ level mask, the accuracy went down, but remained highly significant. Overall accuracy was $84.2 \%$, with specificity and sensitivity of $84.2 \%$ (correctly classifying 16 out of 19 subjects in each group). Follow-up permutation testing (with 2000 repetitions) confirmed that both the overall accuracy $(p=0.005)$ and the class accuracies ( $p=0.005 ; p=0.005)$ were significantly above chance. Therefore, ACF b values from this part of brain alone

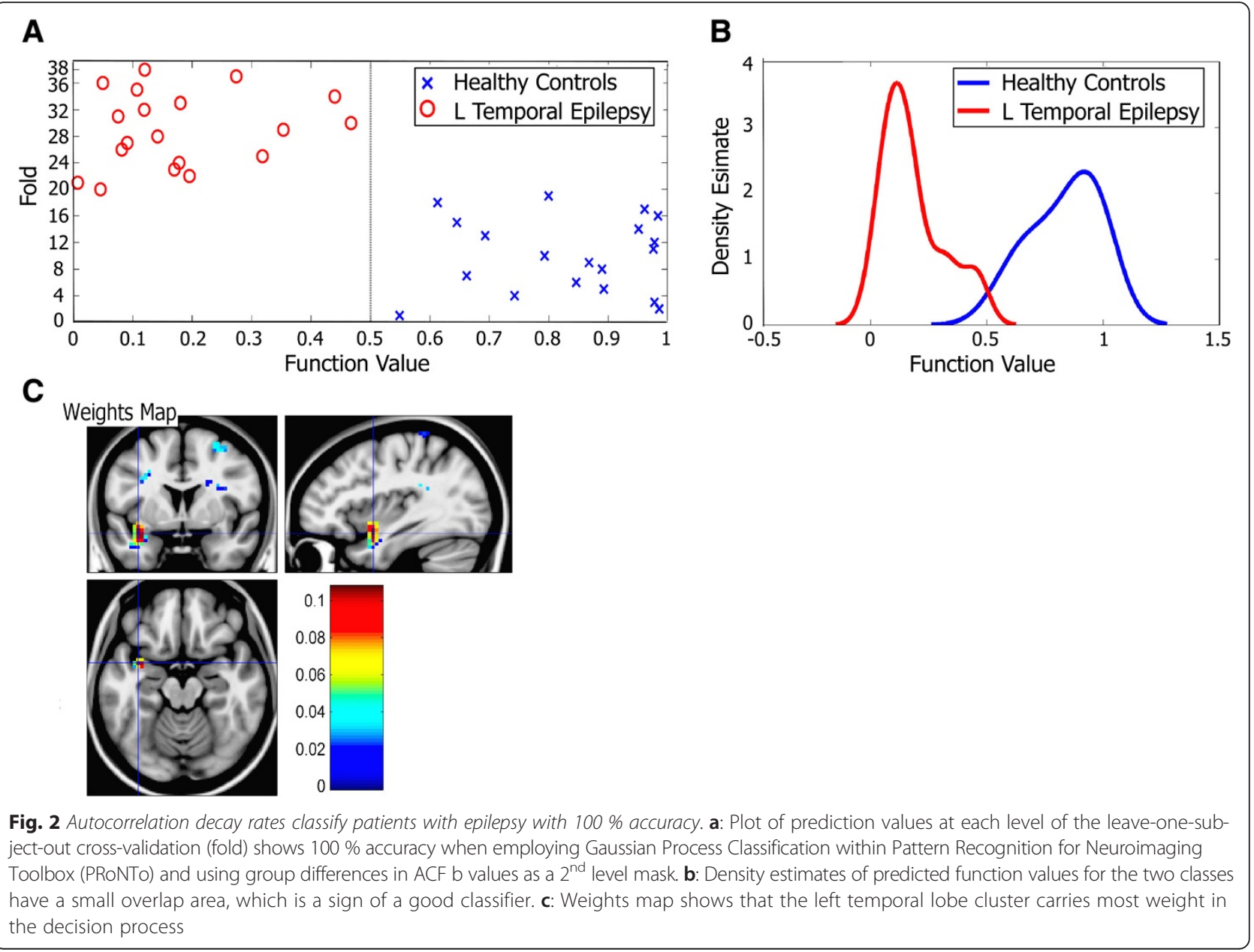


contain sufficient information for significant discrimination between the two subject groups.

As a control, we repeated the classification using the entire grey matter mask as a $2^{\text {nd }}$ level mask. Gray matter mask was obtained from SPM8 and thresholded at 0.5. This resulted in reduced specificity of $53 \%$, not significantly greater than the one expected by chance. This confirms that we discarded most of irrelevant information when constraining the analyses to either of the two $2^{\text {nd }}$ level masks described above.

\section{Constrained dynamics are linked with locally dense/long-} range sparse connectivity

Seed-to-voxel functional connectivity analysis revealed that LTLE patients show increase in connectivity of the most dynamically constrained cluster (ACF-identified cluster at MNI (-36 2-17)) with neighboring left temporal and frontal regions, as well as decreases in connectivity of this cluster with parts of bilateral dorsal posterior cingulate cortex (PCC), contralateral inferior temporal gyrus, and contralateral thalamus, when compared to healthy controls ( $p<0.005$ uncorrected; cluster extent $>10$ voxels), as shown in Fig. 3. Table 3 contains a complete list of significant seed-to-voxel connectivity differences.

Connectivity abnormalities correlate with epilepsy duration In linking neural and clinical features, for the latter we focused on duration rather than seizure frequency since our patient sample did not provide significant variance in the latter. We found a significant positive linear correlation (Pearson's $r=0.61, p=0.005$ ) between the mean connectivity of the cluster in PCC with the ACF-identified seed in left temporal pole and the duration of epilepsy. Therefore, while PCC exhibited overall decrease in connectivity with the seed in patients relative to controls, patients with longer duration of epilepsy had increased connectivity. On the other hand, for the cluster within the contralateral inferior temporal gyrus, there was a clear, but non-significant, trend towards negative correlation of this area's connectivity with the seed and duration of epilepsy (Pearson's $\mathrm{r}=-0.44, p=0.060$ ). For other areas that showed abnormal connectivity to the ACFidentified seed, connectivity with the seed did not exhibit linear relation to epilepsy duration.

Connectivity abnormalities correlate with verbal memory task performance

We found that disrupted local connectivity of the left temporal lobe ACF cluster correlated negatively with performance on the LM-DR task (Pearson's $\mathrm{r}=-0.50$, $p=0.03$, Fig. 4); that is, patients who performed poorly on the verbal memory task exhibited higher local connectivity relative to those who did well. Greater severity of clinical symptoms was thus associated with higher local connectivity.

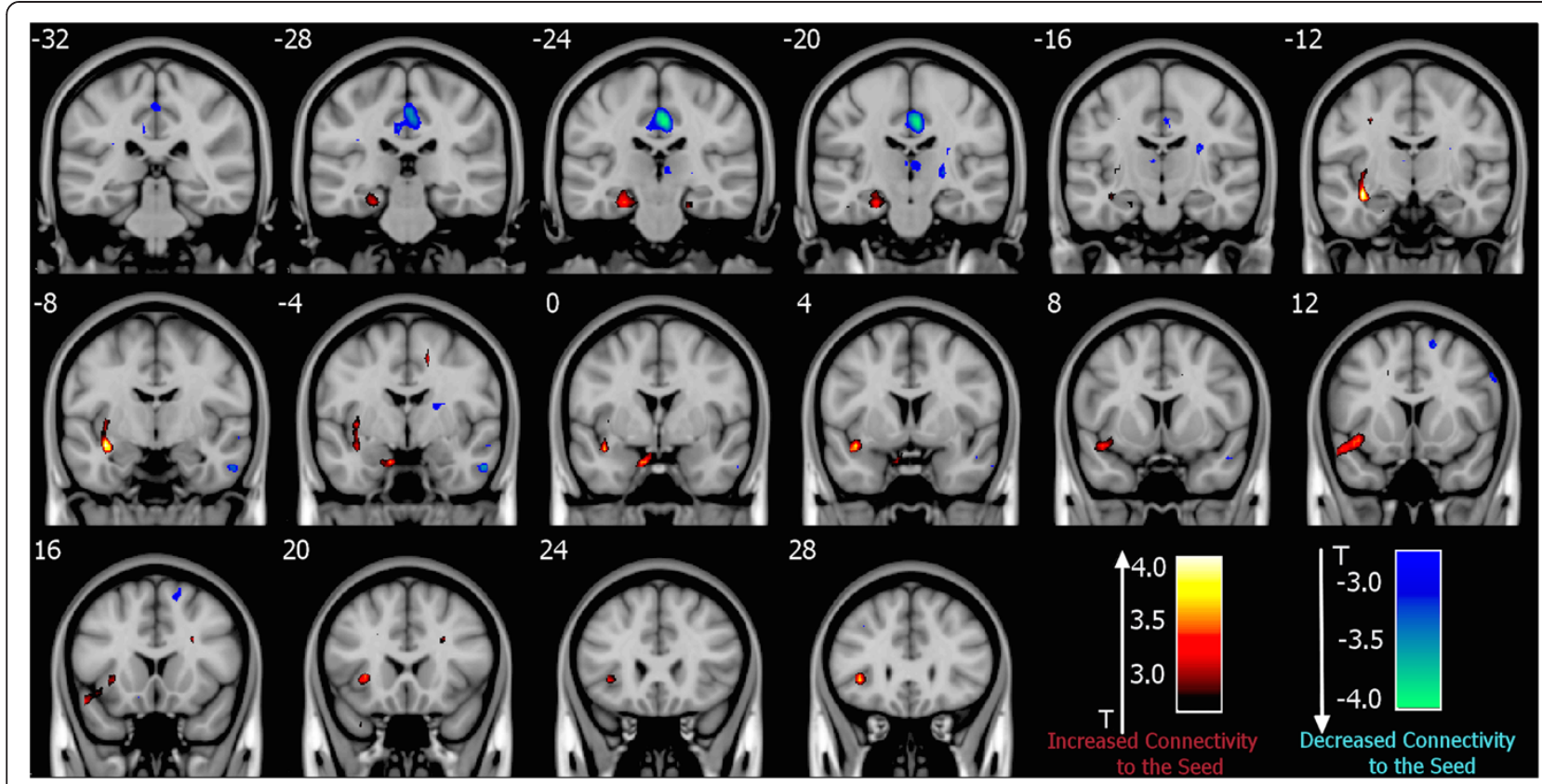

Fig. 3 Seed-to-voxel connectivity of the ACF-identified left temporal lobe cluster is significantly different in patients. Relative to healthy controls, increases in connectivity are observed towards neighboring regions, while decreases are observed towards posterior cingulate cortex, thalamus, and the contralateral temporal lobe 
Table 3 List of regions showing significantly different functional connectivity to the ACF-identified left temporal pole cluster

\begin{tabular}{|c|c|c|c|c|}
\hline & \multicolumn{4}{|c|}{ Left TLE $>$ healthy controls } \\
\hline & Peak MNI & Location description & Cluster extent & t-test (peak level T (p)) \\
\hline \multirow[t]{3}{*}{1} & {$[-36,-10,-14]$} & L Temporopolar Area (BA 38), Hippocampus, & 115 & $4.23\left(7.70 \times 10^{-5}\right)$ \\
\hline & & Inferior Prefrontal Gyrus (BA 47), & & \\
\hline & & Insular Cortex (BA 13) & & \\
\hline \multirow[t]{2}{*}{2} & {$[-24,-25,-20]$} & L Perirhinal Cortex (BA 35), & 40 & $3.34\left(9.88 \times 10^{-4}\right)$ \\
\hline & & Parahippocampal Cortex (BA 36) & & \\
\hline 3 & {$[-36,29,-2]$} & L Inferior Prefrontal Gyrus (BA 47) & 27 & $3.67\left(3.93 \times 10^{-4}\right)$ \\
\hline 4 & {$[-33,-91,-2]$} & L Secondary Visual Cortex (BA 18) & 19 & $3.35\left(9.57 \times 10^{-4}\right)$ \\
\hline \multirow[t]{3}{*}{5} & {$[-27,-67,31]$} & L Associative Visual Cortex (BA 19) & 18 & $3.27\left(1.20 \times 10^{-3}\right)$ \\
\hline & \multicolumn{4}{|c|}{ Left TLE $<$ Healthy Controls } \\
\hline & Peak MNI & Location description & Cluster extent & t-test (peak level T (p)) \\
\hline 1 & {$[6,-22,40]$} & $\begin{array}{l}\text { Bilateral Dorsal Posterior Cingulate Cortex (BA 31), } \\
\text { L Ventral Anterior Cingulate Cortex (BA 24) }\end{array}$ & 97 & $-4.13\left(1.04 \times 10^{-4}\right)$ \\
\hline 2 & {$[54,-7,-29]$} & R Inferior Temporal Gyrus (BA 20) & 12 & $-3.61\left(4.58 \times 10^{-4}\right)$ \\
\hline 3 & {$[15,14,58]$} & R Premotor Cortex (BA 6) & 11 & $-3.36\left(9.27 \times 10^{-4}\right)$ \\
\hline 4 & {$[6,-22,4]$} & R Thalamus & 11 & $-3.11\left(1.80 \times 10^{-3}\right)$ \\
\hline
\end{tabular}

Two-sample $t$-test was performed in CONN Toolbox $(p<0.005, \mathrm{k} \geq 10$ voxels)

\section{Clusters with constrained dynamics form a less efficient network in patients}

Global efficiency of the 16-node network was significantly decreased in patients compared to controls, with one-sided 2 -sample $t$-test $p<0.05$ when varying over costs of $0.20,0.25$, and 0.3 (corresponding to 20, 25, and $30 \%$ of strongest connections kept; $p=0.011$, $p=0.043$, and $p=0.020$, respectively). By thresholding at a certain cost we are in effect fixing the average degree of the network, so that the differences in graph measures presumably reflect changes in network topology. Nevertheless, it is important to note that there is no reliable and fully unbiased way of comparing networks, and that thresholding in this manner may convert non-significant values into edges for networks with low overall connectivity, and discard a number of significant connections for networks with high overall connectivity [60]. We did not find significant between-group differences in other graph metrics tested.
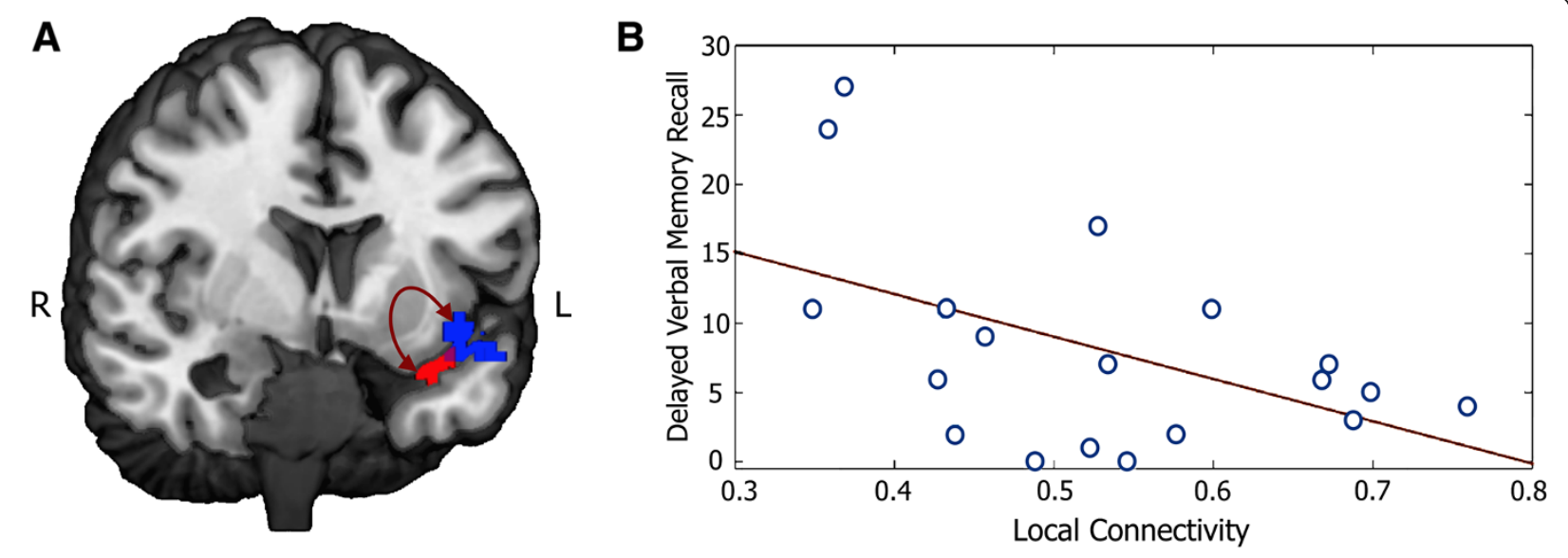

Fig. 4 Abnormalities in functional connectivity correlate with patients'scores on the Logical Memory Delayed Recall task. a: Connectivity of the dysregulated left temporal pole cluster (red) is increased with the neighboring temporopolar/insular/hippocampal region (blue) in patients compared to controls (Table $\mathbf{3}$, Cluster 1). b: The strength of this local connection correlates negatively with patients' performance on the verbal memory task $(r=-0.50, p=0.030, N=19)$ 


\section{Discussion}

We found that patients with left TLE show slower timeseries autocorrelation decay times in the left anterior temporal pole (proximal to the presumed epileptogenic zone) relative to decay times from the same region in healthy controls, indicating loss of complexity and more constrained functional dynamics. Decay time of the ACF discriminated successfully between patients and healthy controls on a subject-by-subjects basis in a purely datadriven manner, achieving accuracy of $100 \%$ in our sample of $N=19$, and therefore showing potential for future validation as a neurodiagnostic tool for localization of epileptic foci.

Changes in BOLD time series complexity were accompanied by changes in local and global connectivity, with increased connectivity with neighboring temporo-frontal areas, and decreased connectivity with regions of the default mode network (DMN) and the contralateral temporal lobe. This result agrees with recent findings that complexity covaries with local connectivity [61] as well as with recent ICA-based findings that MTLE specific networks (which include temporal poles) show increased connectivity in patients, whereas the control-specific network (including thalamus and anterior cingulate cortex) shows decreased connectivity in patients [15].

Complexity of low frequency BOLD fluctuations has been shown to correlate significantly with local connectivity as measured by regional homogeneity (ReHo) [61], implying that increased power spectrum scale invariance $\beta$ (or, analogously, decreased autocorrelation function b values) may be a reflection of enhanced local synchronization. In addition, recent findings suggest that, relative to controls, unilateral MTLE patients show significantly increased regional homogeneity in the ipsilateral parahippocampal gyrus, but also in the midbrain, insula, corpus callosum, bilateral sensorimotor cortex, and frontoparietal subcortical structures [62]. This is in agreement with our findings of decreased $\mathrm{ACF} b$ values in a cluster within the left temporal pole and increased seed-to-voxel connectivity of this cluster towards neighboring areas, as well as with abnormal complexity found in the inferior frontal gyrus, bilateral inferior parietal and postcentral area, and bilateral insula.

The literature often reports structural changes in MTLE, which is most often accompanied by hippocampal sclerosis (HS), but also by (predominantly) ipsilateral atrophy of temporal pole and other temporolimbic structures [63]. It is clinically well established that mesial temporal sclerosis may extend throughout the temporal lobe, and involve the cortex and the white matter, thus leading to extensive temporal lobe atrophy. Increased diffusion rate and decreased anisotropy have been observed in the epileptic focus, of TLE patients with unilateral HS [13, 14]. Increased diffusion rate may be attributed to neuronal necrosis, gliosis, and expanded extracellular space, while the reduction in anisotropy may come from a loss of ordered structure, myelin degradation, and lowered cell density. Structural changes are often non-localizing, and even nonlateralizing, including altered diffusion properties in the contralateral temporal and inferior frontal lobes [14] and widespread significant neocortical thinning in the sensorimotor cortex [64]. Recurrent seizures and structural degeneration may lead to changes in functional connectivity as well: repair mechanisms (such as MMP-9) that degrade the matrix, or metabolic changes that impact glucose utilization, may facilitate local connections while preventing long-range connections.

Our recent simulation studies show that power spectrum scale invariance varies as a function of both input type (excitatory versus inhibitory) and input density, with greater density of inhibitory or lower density of excitatory inputs producing constrained (less complex, more persistent) dynamics [48]. Although epilepsy is often thought of as a hyper-excitatory disorder, this may not necessarily hold true during interictal periods (for example, it has been postulated that ictal epileptic neurophysiological activity can trigger local area neuronal network inhibition in attempt to stabilize the local neuronal network function [65]); constrained dynamics are, in fact, consistent with hubs that contain more inhibitory connections [48]. Furthermore, it has been postulated that epileptogenesis may involve not just the creation of a hyperexcitable state, but also the existence of high connectivity state and non-Markovian recurrent loops [66], in agreement with our finding that epileptogenic regions show higher local connectivity and exhibit longer time series memory (slower autocorrelation decay). The posterior cingulate cortex (PCC) is a pivotal hub for integration and mediation of information in the brain [67]. It has been implicated in a range of functions, and shown to be a part of the Default Mode Network (DMN) and Dorsal Attention Network. It has strong reciprocal connections to mesial temporal lobe memoryrelated structures [68]. Our ACF-identified cluster showed decreases in connectivity with PCC, in agreement with a couple of recent studies showing diminished connectivity between PCC and bilateral mesial temporal structures in left MTLE patients [21, 69]. In addition, our findings suggest that there is a linear relationship between left temporal pole connectivity with PCC and epilepsy duration, over which the connection is restored over time. However, this restoration seems to be accompanied by aggravated decrease in connectivity with the opposite temporal lobe.

The Wechsler Memory Scale is one of the most commonly used memory tests in patients with epilepsy, and is often a part of standard pre-surgical evaluation. Patients with temporal lobe epilepsy have been found to have 
lower scores when compared to healthy controls, but the tests on their own have been unable to lateralize temporal epilepsy successfully [70, 71]. We found that local increase in connectivity of the dynamically constrained cluster in patients correlated negatively with scores on the Logical Memory Delayed Recall task suggesting, as expected, that more severe symptoms were associated with higher local connectivity. Therefore, local connectivity of the left temporal lobe ACF-identified region was not only increased in patients compared to controls, but was also associated with severity of verbal memory impairment within the patient population. This is not surprising considering that the disconnected cluster included parts of affected hippocampus, a structure known to be heavily involved in memory processing.

\section{Limitations and future directions}

The current gold standard for localization of focal regions includes identification of an epileptogenic zone on intracranial EEG recordings combined with postoperative seizure freedom following its resection. Due to invasive nature of intracranial recordings and the extensive temporal duration required to establish seizure freedom with confidence, data of such nature are limited. In this study, we utilized data from nineteen patients with concordant clinical findings, including results of long-term video EEG monitoring and structural MRI abnormalities confirmed by an experienced neurologist. While this group serves as a benchmark for the application of the technique developed here, and comparisons of individual ACF b maps to the mean control map point to clinically relevant region in $100 \%$ of tested subjects, future work will address its applicability in subjects with other forms of epilepsy, ideally with the epileptogenic zone confirmed via invasive recordings. The method presented here is an alternative to multimodal methods such as EEG-fMRI, which has the advantage of eliminating the electrophysiological setups that are incompatible with some MR head-coils, avoids extensive set-up times, is completely fMRI data-driven (avoiding assumptions with respect to the shape of the hemodynamic response function and modeling of epileptic events), and utilizes only seven minutes of resting state data without the necessity of active patient participation. Our aim is to provide a first step towards a non-invasive method that reliably detects focal regions in patients with drug resistant cryptogenic epilepsy.

\section{Conclusions}

We developed techniques for the interictal identification of epileptic foci through complexity and network analyses of fMRI time-series. In a completely non-invasive and data-driven manner, based on complexity values calculated from resting-state fMRI images alone, we were able to achieve $100 \%$ accuracy in distinguishing between
19 healthy controls and 19 epileptic patients with welldefined left temporal epileptic foci. Our method has shown high sensitivity and specificity in localizing focal points, while providing additional information about the underlying dynamics of epileptic brains. Since the method does not explicitly depend on existence of MRI-detectable structural abnormalities, it could eventually be applied to patients with epileptic foci that are inadequately defined or poorly localized based on current state of the art neuroimaging techniques. Functional MRI analytical methods that target network dynamics therefore hold promise in providing novel and clinically valuable tools for the identification and resection of epileptogenic foci.

Competing interests

The authors declare that they have no competing interests.

\section{Authors' contributions}

SN participated in the design of the study, conducted the analyses, and wrote the manuscript. LRMP conceived and coordinated the design of the study, and wrote the manuscript. SS participated in the design of the study and helped to draft the manuscript. CR and ACS participated in the fMRI data collection. TRV participated in the clinical assessment of the epilepsy patients, and interpretation of the results. JPL helped with the design and coordination of the study. ACG collected the clinical information and performed the cognitive performance assessment. JSI conceived and coordinated the design of the study and the data collection, and wrote the manuscript. All authors read and approved the final manuscript.

\section{Acknowledgements \\ This research was supported by the National Science Foundation (CBET 1264440; LRMP) and the Sao Paulo Research Foundation (Young Investigators Awards FAPESP 11/08573-4; JSI).}

\section{Author details}

${ }^{1}$ Department of Biomedical Engineering, Stony Brook University School of Medicine, Stony Brook, NY 11794, USA. ²Department of Radiology, A. A. Martinos Center for Biomedical Imaging, Massachusetts General Hospital, Charlestown, MA 02129, USA. ${ }^{3}$ Department of Neurosciences and Behavior, University of Sao Paulo (USP), Ribeirao Preto, SP 14049, Brazil. ${ }^{4}$ Department of Science and Technology, Federal University of Sao Paulo, Sao Jose dos Campos, SP 12231, Brazil.

Received: 13 April 2015 Accepted: 4 December 2015

Published online: 21 December 2015

\section{References}

1. Banerjee PN, Filippi D, Allen HW. The descriptive epidemiology of epilepsy-a review. Epilepsy Res. 2009;85(1):31-45. doi:10.1016/j.eplepsyres.2009.03.003.

2. Lehnertz K, Bialonski S, Horstmann MT, Krug D, Rothkegel A, Staniek M, et al. Synchronization phenomena in human epileptic brain networks. J Neurosci Methods. 2009;183(1):42-8. doi:10.1016/j.jneumeth.2009.05.015.

3. Kwan P, Brodie MJ. Early identification of refractory epilepsy. N Engl J Med. 2000;342(5):314-9. doi:10.1056/NEJM200002033420503.

4. Tellez-Zenteno JF, Dhar R, Wiebe S. Long-term seizure outcomes following epilepsy surgery: a systematic review and meta-analysis. Brain : a journal of neurology. 2005;128(Pt 5):1188-98. doi:10.1093/brain/awh449.

5. Wellmer J, von der Groeben F, Klarmann U, Weber C, Elger CE, Urbach H, et al. Risks and benefits of invasive epilepsy surgery workup with implanted subdural and depth electrodes. Epilepsia. 2012;53(8):1322-32. doi:10.1111/j. 1528-1167.2012.03545.x.

6. Klamer S, Rona S, Elshahabi A, Lerche H, Braun C, Honegger J, et al. Multimodal effective connectivity analysis reveals seizure focus and propagation in musicogenic epilepsy. Neurolmage. 2015;113:70-7. doi:10.1016/j.neuroimage.2015.03.027.

7. Vaudano AE, Avanzini P, Tassi L, Ruggieri A, Cantalupo G, Benuzzi F, et al. Causality within the epileptic network: an EEG-fMRI study validated by intracranial EEG. Front Neurol. 2013;4:185. doi:10.3389/fneur.2013.00185. 
8. Grouiller F, Thornton RC, Groening K, Spinelli L, Duncan JS, Schaller K, et al. With or without spikes: localization of focal epileptic activity by simultaneous electroencephalography and functional magnetic resonance imaging. Brain : a journal of neurology. 2011;134(Pt 10):2867-86. doi:10.1093/brain/awr156.

9. Bagshaw AP, Aghakhani Y, Benar CG, Kobayashi E, Hawco C, Dubeau F, et al. EEG-fMRI of focal epileptic spikes: analysis with multiple haemodynamic functions and comparison with gadolinium-enhanced MR angiograms. Hum Brain Mapp. 2004;22(3):179-92. doi:10.1002/hbm.20024.

10. Elshoff L, Groening K, Grouiller F, Wiegand G, Wolff S, Michel C, et al. The value of EEG-fMRI and EEG source analysis in the presurgical setup of children with refractory focal epilepsy. Epilepsia. 2012;53(9):1597-606. doi:10.1111/j. 1528-1167.2012.03587.x.

11. Grouiller F, Delattre BM, Pittau F, Heinzer S, Lazeyras F, Spinelli L, et al. All-in-one interictal presurgical imaging in patients with epilepsy: singlesession EEG/PET/(f)MRI. Eur J Nucl Med Mol Imaging. 2015;42(7):1133-43. doi:10.1007/s00259-015-3045-2.

12. Hogan RE, Kaiboriboon K, Bertrand ME, Rao V, Acharya J. Composite SISCOM perfusion patterns in right and left temporal seizures. Arch Neurol. 2006:63(10):1419-26. doi:10.1001/archneur.63.10.1419.

13. Assaf BA, Mohamed FB, Abou-Khaled KJ, Williams JM, Yazeji MS, Haselgrove $J$, et al. Diffusion tensor imaging of the hippocampal formation in temporal lobe epilepsy. AJNR American journal of neuroradiology. 2003;24(9):1857-62.

14. Focke NK, Yogarajah M, Bonelli SB, Bartlett PA, Symms MR, Duncan JS. Voxel-based diffusion tensor imaging in patients with mesial temporal lobe epilepsy and hippocampal sclerosis. Neurolmage. 2008;40(2):728-37. doi:10.1016/j.neuroimage.2007.12.031.

15. Maneshi M, Vahdat S, Fahoum F, Grova C, Gotman J. Specific resting-state brain networks in mesial temporal lobe epilepsy. Front Neurol. 2014;5:127. doi:10.3389/fneur.2014.00127.

16. Pittau F, Grova C, Moeller F, Dubeau F, Gotman J. Patterns of altered functional connectivity in mesial temporal lobe epilepsy. Epilepsia. 2012;53(6):1013-23. doi:10.1111/j.1528-1167.2012.03464.x.

17. Pereira FR, Alessio A, Sercheli MS, Pedro T, Bilevicius E, Rondina JM, et al. Asymmetrical hippocampal connectivity in mesial temporal lobe epilepsy: evidence from resting state fMRI. BMC Neurosci. 2010;11:66. doi:10.1186/ 1471-2202-11-66.

18. Zhang Z, Lu G, Zhong Y, Tan Q, Liao W, Chen Z, et al. Impaired perceptual networks in temporal lobe epilepsy revealed by resting fMRI. J Neurol. 2009;256(10):1705-13. doi:10.1007/s00415-009-5187-2.

19. Zhang Z, Lu G, Zhong Y, Tan Q, Liao W, Wang Z, et al. Altered spontaneous neuronal activity of the default-mode network in mesial temporal lobe epilepsy. Brain Res. 2010;1323:152-60. doi:10.1016/j.brainres.2010.01.042.

20. Liao W, Zhang Z, Pan Z, Mantini D, Ding J, Duan X, et al. Altered functional connectivity and small-world in mesial temporal lobe epilepsy. PLoS One. 2010;5(1), e8525. doi:10.1371/journal.pone.0008525.

21. Liao W, Zhang Z, Pan Z, Mantini D, Ding J, Duan X, et al. Default mode network abnormalities in mesial temporal lobe epilepsy: a study combining fMRI and DTI. Hum Brain Mapp. 2011;32(6):883-95. doi:10.1002/hbm.21076.

22. Bettus G, Guedj E, Joyeux F, Confort-Gouny S, Soulier E, Laguitton V, et al. Decreased basal fMRI functional connectivity in epileptogenic networks and contralateral compensatory mechanisms. Hum Brain Mapp. 2009;30(5):1580-91.

23. Bettus G, Bartolomei F, Confort-Gouny S, Guedj E, Chauvel P, Cozzone PJ, et al. Role of resting state functional connectivity MRI in presurgical investigation of mesial temporal lobe epilepsy. J Neurol Neurosurg Psychiatry. 2010;81(10):1147-54. doi:10.1136/jnnp.2009.191460.

24. Tracy Jl, Doucet GE. Resting-state functional connectivity in epilepsy: growing relevance for clinical decision making. Curr Opin Neurol. 2015;28(2):158-65. doi:10.1097/WCO.0000000000000178.

25. Bullmore E, Sporns O. Complex brain networks: graph theoretical analysis of structural and functional systems. Nat Rev Neurosci. 2009;10(3):186-98. doi:10.1038/nrn2575.

26. Horstmann MT, Bialonski S, Noennig N, Mai H, Prusseit J, Wellmer J, et al State dependent properties of epileptic brain networks: comparative graph-theoretical analyses of simultaneously recorded EEG and MEG. Clin Neurophysiol. 2010;121(2):172-85. doi:10.1016/j.clinph.2009.10.013.

27. Bartolomei F, Bettus G, Stam CJ, Guye M. Interictal network properties in mesial temporal lobe epilepsy: a graph theoretical study from intracerebral recordings. Clin Neurophysiol. 2013;124(12):2345-53. doi:10.1016/j.clinph. 2013.06.003

28. Stam CJ. Modern network science of neurological disorders. Nat Rev Neurosci. 2014;15(10):683-95. doi:10.1038/nrn3801.
29. Morgan VL, Rogers BP, Sonmezturk HH, Gore JC, Abou-Khalil B. Cross hippocampal influence in mesial temporal lobe epilepsy measured with high temporal resolution functional magnetic resonance imaging. Epilepsia. 2011;52(9):1741-9. doi:10.1111/j.1528-1167.2011.03196.x.

30. Maccotta L, He BJ, Snyder AZ, Eisenman LN, Benzinger TL, Ances BM, et al. Impaired and facilitated functional networks in temporal lobe epilepsy. Neurolmage Clinical. 2013;2:862-72. doi:10.1016/j.nicl.2013.06.011.

31. Stufflebeam SM, Liu H, Sepulcre J, Tanaka N, Buckner RL, Madsen JR. Localization of focal epileptic discharges using functional connectivity magnetic resonance imaging. J Neurosurg. 2011;114(6):1693-7. doi:10.3171/2011.1.JNS10482.

32. Lowen SB, Cash SS, Poo M, Teich MC. Quantal neurotransmitter secretion rate exhibits fractal behavior. The Journal of neuroscience : the official journal of the Society for Neuroscience. 1997;17(15):5666-77.

33. Levina A, Herrmann JM, Geisel T. Dynamical synapses causing self-organized criticality in neural networks. Nat Phys. 2007;3(12):857-60. doi:10.1038/Nphys758.

34. Rubinov M, Sporns O, Thivierge JP, Breakspear M. Neurobiologically realistic determinants of self-organized criticality in networks of spiking neurons. PLoS Comput Biol. 2011;7(6), e1002038. doi:10.1371/journal.pcbi.1002038.

35. Bedard C, Destexhe A. Macroscopic models of local field potentials and the apparent 1/f noise in brain activity. Biophys J. 2009;96(7):2589-603. doi:10.1016/j.bpj.2008.12.3951.

36. He BJ, Raichle ME. The fMRI signal, slow cortical potential and consciousness. Trends Cogn Sci. 2009;13(7):302-9. doi:10.1016/j.tics.2009.04.004.

37. Lai MC, Lombardo MV, Chakrabarti B, Sadek SA, Pasco G, Wheelwright SJ, et al. A shift to randomness of brain oscillations in people with autism. Biol Psychiatry. 2010;68(12):1092-9. doi:10.1016/j.biopsych.2010.06.027.

38. Freyer F, Aquino K, Robinson PA, Ritter P, Breakspear M. Bistability and non-Gaussian fluctuations in spontaneous cortical activity. The Journal of neuroscience : the official journal of the Society for Neuroscience. 2009:29(26):8512-24. doi:10.1523/JNEUROSCI.0754-09.2009.

39. Maxim V, Sendur L, Fadili J, Suckling J, Gould R, Howard R, et al. Fractional Gaussian noise, functional MRI and Alzheimer's disease. Neurolmage. 2005;25(1):141-58. doi:10.1016/j.neuroimage.2004.10.044.

40. Radulescu AR, Rubin D, Strey HH, Mujica-Parodi LR. Power spectrum scale invariance identifies prefrontal dysregulation in paranoid schizophrenia. Hum Brain Mapp. 2012;33(7):1582-93. doi:10.1002/hbm.21309.

41. Wilczynski GM, Konopacki FA, Wilczek E, Lasiecka Z, Gorlewicz A, Michaluk P, et al. Important role of matrix metalloproteinase 9 in epileptogenesis. J Cell Biol. 2008;180(5):1021-35. doi:10.1083/jcb.200708213.

42. Monto S, Vanhatalo S, Holmes MD, Palva JM. Epileptogenic neocortical networks are revealed by abnormal temporal dynamics in seizure-free subdural EEG. Cereb Cortex. 2007;17(6):1386-93. doi:10.1093/cercor/bhl049.

43. Bruzzo AA, Gesierich B, Santi M, Tassinari CA, Birbaumer N, Rubboli G. Permutation entropy to detect vigilance changes and preictal states from scalp EEG in epileptic patients. A preliminary study. Neurol Sci. 2008;29(1):3-9. doi:10.1007/s10072-008-0851-3.

44. Molteni E, Perego P, Zanotta N, Reni G. Entropy analysis on EEG signal in a case study of focal myoclonus. leee Eng Med Bio. 2008:4724-7. doi:10.1109/ lembs.2008.4650268.

45. Protzner AB, Valiante TA, Kovacevic N, McCormick C, McAndrews MP. Hippocampal signal complexity in mesial temporal lobe epilepsy: a noisy brain is a healthy brain. Arch Ital Biol. 2010;148(3):289-97.

46. Helmstaedter C, Kurthen M, Lux S, Reuber M, Elger CE. Chronic epilepsy and cognition: a longitudinal study in temporal lobe epilepsy. Ann Neurol. 2003; 54(4):425-32. doi:10.1002/ana.10692.

47. Wechsler D. Wechsler Memory Scale-Revised manual. San Antonio, TX: Psychological Corporation; 1987.

48. Radulescu A, Mujica-Parodi LR. Network connectivity modulates power spectrum scale invariance. Neurolmage. 2014;90:436-48. doi:10.1016/j. neuroimage.2013.12.001

49. Tolkunov D, Rubin D, Mujica-Parodi L. Power spectrum scale invariance quantifies limbic dysregulation in trait anxious adults using fMRl: adapting methods optimized for characterizing autonomic dysregulation to neural dynamic time series. Neurolmage. 2010;50(1):72-80. doi:10.1016/j. neuroimage.2009.12.021

50. He BJ. Scale-free properties of the functional magnetic resonance imaging signal during rest and task. The Journal of neuroscience : the official journal of the Society for Neuroscience. 2011;31(39):13786-95. doi:10.1523/ JNEUROSCI.2111-11.2011.

51. Clauset A, Shalizi CR, Newman MEJ. Power-law distributions in empirical data. Siam Rev. 2009;51(4):661-703. doi:10.1137/070710111. 
52. Power JD, Barnes KA, Snyder AZ, Schlaggar BL, Petersen SE. Spurious but systematic correlations in functional connectivity MRI networks arise from subject motion. Neurolmage. 2012;59(3):2142-54. doi:10.1016/j.neuroimage. 2011.10.018.

53. Biswal B, Yetkin FZ, Haughton VM, Hyde JS. Functional connectivity in the motor cortex of resting human brain using echo-planar MRI. Magn Reson Med. 1995;34(4):537-41.

54. Biswal BB, Van Kylen J, Hyde JS. Simultaneous assessment of flow and BOLD signals in resting-state functional connectivity maps. NMR Biomed. 1997; 10(4-5):165-70.

55. Miezin FM, Maccotta L, Ollinger JM, Petersen SE, Buckner RL. Characterizing the hemodynamic response: effects of presentation rate, sampling procedure, and the possibility of ordering brain activity based on relative timing. Neurolmage. 2000;11(6 Pt 1):735-59. doi:10.1006/nimg.2000.0568.

56. Song XW, Dong ZY, Long XY, Li SF, Zuo XN, Zhu CZ, et al. REST: a toolkit for resting-state functional magnetic resonance imaging data processing. PLoS One. 2011:6(9), e25031. doi:10.1371/journal.pone.0025031.

57. Schrouff J, Rosa MJ, Rondina JM, Marquand AF, Chu C, Ashburner J, et al. PRoNTo: pattern recognition for neuroimaging toolbox. Neuroinformatics. 2013;11(3):319-37. doi:10.1007/s12021-013-9178-1.

58. Latora V, Marchiori M. Efficient behavior of small-world networks. Phys Rev Lett. 2001:87(19):198701.

59. Achard S, Bullmore E. Efficiency and cost of economical brain functional networks. PLoS Comput Biol. 2007;3(2), e17. doi:10.1371/journal.pcbi.0030017.

60. van Wijk BC, Stam CJ, Daffertshofer A. Comparing brain networks of different size and connectivity density using graph theory. PLoS One. 2010;5(10), e13701. doi:10.1371/journal.pone.0013701.

61. Anderson JS, Zielinski BA, Nielsen JA, Ferguson MA. Complexity of lowfrequency blood oxygen level-dependent fluctuations covaries with local connectivity. Hum Brain Mapp. 2014;35(4):1273-83. doi:10.1002/hbm.22251.

62. Zeng H, Pizarro R, Nair VA, La C, Prabhakaran V. Alterations in regional homogeneity of resting-state brain activity in mesial temporal lobe epilepsy. Epilepsia. 2013;54(4):658-66. doi:10.1111/epi.12066.

63. Coste S, Ryvlin P, Hermier M, Ostrowsky K, Adeleine P, Froment JC, et al. Temporopolar changes in temporal lobe epilepsy: a quantitative MRI-based study. Neurology. 2002;59(6):855-61.

64. Labate A, Cerasa A, Aguglia U, Mumoli L, Quattrone A, Gambardella A. Neocortical thinning in "benign" mesial temporal lobe epilepsy. Epilepsia. 2011;52(4):712-7. doi:10.1111/j.1528-1167.2011.03038.x.

65. Tebartz van Elst L, Krishnamoorthy ES, Schulze-Bonhage A, Altenmuller DM, Richter H, Ebert D, et al. Local area network inhibition: a model of a potentially important paraepileptic pathomechanism in neuropsychiatric disorders. Epilepsy \& behavior : E\&B. 2011;22(2):231-9. doi:10.1016/j.yebeh.2011.06.016.

66. Hsu D, Tang A, Hsu M, Beggs JM. Simple spontaneously active Hebbian learning model: homeostasis of activity and connectivity, and consequences for learning and epileptogenesis. Phys Rev E Stat Nonlinear Soft Matter Phys. 2007;76(4 Pt 1):041909.

67. van den Heuvel MP, Sporns O. Rich-club organization of the human connectome. The Journal of neuroscience : the official journal of the Society for Neuroscience. 2011:31(44):15775-86. doi:10.1523/JNEUROSCI.3539-11.2011.

68. Maddock RJ, Garrett AS, Buonocore MH. Remembering familiar people: the posterior cingulate cortex and autobiographical memory retrieval. Neuroscience. 2001;104(3):667-76.

69. James GA, Tripathi SP, Ojemann JG, Gross RE, Drane DL. Diminished default mode network recruitment of the hippocampus and parahippocampus in temporal lobe epilepsy. J Neurosurg. 2013;119(2):288-300. doi:10.3171/2013. 3.JNS121041.

70. Helmstaedter C, Wietzke J, Lutz MT. Unique and shared validity of the "Wechsler logical memory test", the "California verbal learning test", and the "verbal learning and memory test" in patients with epilepsy. Epilepsy Res. 2009;87(2-3):203-12. doi:10.1016/j.eplepsyres.2009.09.002.

71. Soble JR, Eichstaedt KE, Waseem H, Mattingly ML, Benbadis SR, Bozorg AM et al. Clinical utility of the Wechsler memory scale-fourth edition (WMS-IV) in predicting laterality of temporal lobe epilepsy among surgical candidates. Epilepsy \& behavior : E\&B. 2014;41:232-7. doi:10.1016/j.yebeh.2014.10.014.

\section{Submit your next manuscript to BioMed Central and we will help you at every step:}

- We accept pre-submission inquiries

- Our selector tool helps you to find the most relevant journal

- We provide round the clock customer support

- Convenient online submission

- Thorough peer review

- Inclusion in PubMed and all major indexing services

- Maximum visibility for your research

Submit your manuscript at www.biomedcentral.com/submit
Biomed Central 